\title{
Asociación entre tuberculosis y diabetes mellitus en la Región Metropolitana
}

\author{
TANIA HERRERA M.*, ELIZABETH LEIVA Z.**, FÉLIX MARTÍN H.**, \\ MELISA MIRANDA O.** y CAROLINA MORALES O.**
}

\section{Associated tuberculosis and diabetes mellitus in Santiago de Chile Metropolitan area}

Background: Diabetes mellitus (DM) is recognized as an important risk factor to tuberculosis (TB). However, there is significant diabetes under registration among tuberculosis cases which does not allow quantify the magnitude of this group. This study aims to determine the prevalence of DM among $T B$ cases diagnosed in 2012 in Santiago de Chile, metropolitan area and to estimate the association of these two diseases. Method: A cross sectional study was undertaken. TB cases reported in the National Register of Tuberculosis were sought in the Information System for Management of Health Guarantees (SIGGES) to determine we ther or not they had be sides DM. The analysis of prevalence by age, sex and health service area was carried out, prevalence ratio was used to determine the level of association, and incidence rate of tuberculosis among diabetics and non-diabetics was estimated. Results: 23 TB cases were excluded for lack of necessary information, leaving a total of 821 cases. Prevalence of DM was $15.6 \%$ (13.2-18.2), with a prevalence ratio adjusted by age of 1.29 (1.28-1.29) respect to general population, and 1.73 (1.72-1.73) adjusted by sex. The estimated incidence rate of TB among diabetic population is 24.3 per 100,000, that is to say 1.7 times higher than general population of the region. Conclusions: Prevalence of DM in tuberculosis cases in Metropolitan Region was greater than in the general population. The study confirms that diabetics are a risk group for developing tuberculosis.

Key words: Tuberculosis, Diabetes Mellitus, Risk Groups.

\section{Resumen}

Introducción: Los pacientes con diabetes mellitus (DM) son reconocidos como grupo de riesgo para desarrollar tuberculosis. Sin embargo, existe un importante sub-registro de la diabetes como factor de riesgo entre los casos de tuberculosis, que no permite cuantificar la magnitud de este grupo. Este estudio busca determinar la prevalencia de DM entre los casos de tuberculosis (TB) diagnosticados el año 2012 en la Región Metropolitana y estimar la asociación entre estas dos patologías. Método: Se efectuó un estudio transversal analítico, en que todos los casos de TB mayores de 15 años de la Región Metropolitana del año 2012 consignados en el Registro Nacional de Tuberculosis, fueron buscados en el Sistema de Información para la Gestión de Garantías en Salud (SIGGES) para determinar si tenían o no DM. Se realizó el análisis de prevalencias por edad, sexo y Servicio de Salud y se determinó el nivel de asociación con la razón de prevalencia y la estimación de la tasa de incidencia de tuberculosis entre diabéticos y no diabéticos. Resultados: Del total de casos de TB de la región Metropolitana se excluyeron 23 casos por no disponer de la información necesaria, quedando un total de 821 casos. La prevalencia de DM fue de 15,6\% (13,2-18,2), con una razón de prevalencia ajustada por edad de 1,29 (1,28-1,29) respecto a la población general y de 1,73 (1,72-1,73) ajustada por sexo. La tasa de incidencia estimada de TB entre población diabética es de 24,3 por 100.000, 1,7 veces la de la población general de la región. Conclusiones: La prevalencia de DM en la población con tuberculosis de la Región Metropolitana fue mayor que la encontrada en la población general y se ratifica a los diabéticos como grupo de riesgo para desarrollar tuberculosis.

Palabras clave: Tuberculosis, Diabetes mellitus, Grupos de Riesgo.

\footnotetext{
* Médico Salubrista. Directora del Programa de Control y Eliminación de la Tuberculosis.

** Estudiante de Medicina. Universidad de Chile.
} 


\section{Introducción}

Si bien Chile pertenece al grupo de países de baja prevalencia de tuberculosis en Latinoamérica, presentando tasas de incidencia menores a 20 por 100.000 habitantes desde el año 2000 , la tuberculosis (TB) continúa siendo un importante problema de salud pública. La meta planteada para el año 2010 de avanzar hacia la "Fase de Eliminación Avanzada", es decir, alcanzar una incidencia inferior a 10 por 100.000 habitantes, no fue lograda, manteniéndose una incidencia de 13 por 100.000 desde el año $2009^{1}$.

El estancamiento observado en el descenso de la incidencia de TB representa una amenaza al cumplimiento de la meta sanitaria planteada en los "Objetivos sanitarios de la década 20112020", que corresponde a alcanzar una tasa de incidencia menor a 5 por 100.000 habitantes el año 2020, tasa que corresponde a la eliminación de la tuberculosis como problema de salud pública ${ }^{2}$.

La disminución de la incidencia de TB favorece la concentración de la enfermedad en grupos vulnerables, tanto por la presencia de factores de riesgo biológicos como sociodemográficos ${ }^{3}$. El Programa de Control y Eliminación de la Tuberculosis (PROCET) ha identificado a las personas que sufren de Diabetes Mellitus (DM) como uno de los grupos con riesgo aumentado de padecer de tuberculosis ${ }^{4}$.

La importancia de la relación entre TB y DM ya ha sido establecida por la OMS. Sus informes indican que la población diabética estaría expuesta a un riesgo 2 a 3 veces mayor de desarrollar TB y que alrededor del $10 \%$ de los casos de TB mundial serían diabéticos 5 .

Por otra parte, la DM es una patología que ha ido en aumento en nuestro país, aumentando su prevalencia en población mayor de 15 años desde $7,5 \%$ el año 2006 a 9,4\% (IC95\% 8,1-10,9) el $2010^{6,7}$. En la Región Metropolitana, que representa el $40 \%$ de la población de Chile, la prevalencia de DM según la Encuesta Nacional de Salud 2010 (ENS) fue de 9,2\% (IC no publicado) ${ }^{7}$. Al mismo tiempo esta región abarca en términos absolutos una gran cantidad de los enfermos de tuberculosis del país ${ }^{3}$.

A pesar que el PROCET recoge desde el año 2008 la presencia de factores de riesgo asociados a TB en forma sistemática ${ }^{3}$, existe un importante sub registro de la presencia de DM entre los casos de $\mathrm{TB}$, que no permite conocer la magnitud que tiene este grupo de riesgo en Chile, ni estimar la relación entre ambas patologías ${ }^{8}$. El presente estudio busca establecer la prevalencia de DM entre los casos nuevos de TB mayores de 15 años de la Región Metropolitana registrados durante el año 2012 y determinar la asociación entre ambas patologías con el fin de orientar futuras acciones del Programa de Tuberculosis en este grupo de riesgo.

\section{Metodología}

Se realizó un estudio transversal analítico para determinar la prevalencia de DM entre los casos de TB de la Región Metropolitana y comparar la probabilidad de padecer tuberculosis entre diabéticos y no diabéticos para el año 2012.

Para obtener el dato de la presencia de DM en los casos de TB, se realizó la búsqueda de cada caso nuevo de tuberculosis mayor de 15 años del Registro Nacional de Tuberculosis 2012 en el Sistema de Información para la Gestión de Garantías Explícitas en Salud (SIGGES), sistema informático que permite ubicar a través del RUN* a todos los residentes del país, y que entrega el dato del diagnóstico de DM por tratarse ésta de una patología GES**.

Una vez obtenidos estos datos se caracterizaron los casos de TB con DM en cuanto a su edad, sexo, Servicio de Salud al que pertenecen, localización de la TB y método de confirmación diagnóstica de casos pulmonares. Se calculó la prevalencia de diabéticos entre los casos nuevos de tuberculosis de la Región Metropolitana y por Servicio de Salud.

Para determinar si existe mayor probabilidad de ser diabético entre los enfermos de tuberculosis y la población general, se calculó la razón de prevalencia (RP), comparando la prevalencia de DM en casos nuevos de TB y en población general nacional ajustada por edad y por sexo. Además se calculó la prevalencia de TB por grupo de edad y sexo y se comparó con la reportada por la Encuesta Nacional de Salud.

Finalmente, se estimó la incidencia de TB en la población diabética y no diabética de la región metropolitana.

Los datos personales de los casos en estudio fueron utilizados exclusivamente para la ejecución de la investigación, asegurando su confidencialidad.

\footnotetext{
* Rol Único Nacional. Sistema numerado de identificación individual usado en Chile.

** Garantías Explícitas de Salud: conjunto de problemas de salud priorizados por el país para su control.
} 


\section{Resultados}

Los casos nuevos de TB en mayores de 15 años de la Región Metropolitana para el año 2012 corresponden a 844, de los cuales 23 (2,7\%) fueron excluidos de este estudio debido a RUT omitido o incorrecto que no permitió la búsqueda en el SIGGES, obteniéndose un total de 821 casos.

Dentro de los casos analizados el $61,6 \%$ fueron hombres. La mediana de la edad es de 47 años y recorrido intercuartil de 32- 61 años. El porcentaje de mayores de 65 años fue de $20 \%$.

El total de casos registrados como diabéticos en el SIGGES entre los casos nuevos de TB en mayores de 15 años fue de 128, de ellos el $53,1 \%$ son hombres y $46,9 \%$ mujeres. En cuanto al análisis por edad, la mediana de los casos de TB con DM correspondió a 60 años, recorrido intercuartil 50-70 años. El 0,7\% tiene entre 15 a 24 años, $13,2 \%$ entre 25 y 44 años, $46 \%$ entre 45 y 64 años y $39,8 \%$ tiene 65 o más años.

La distribución por Servicio de Salud es la siguiente: 24\% S. Salud Metropolitano Sur, 19\% S. Salud Metropolitano Suroriente, 18\% S. Salud Metropolitano Occidente, 17\% S. Salud Metropolitano Central, 11\% S. Salud Metropolitano Norte y $11 \% \mathrm{~S}$. Salud Metropolitano Oriente.

El 78,9\% de los casos TB-DM corresponde a TB pulmonar. De ellos, el 77\% se diagnosticó a través de baciloscopía y el $17 \%$ por cultivo. $1 \%$ se diagnosticó por biopsia pulmonar y en 5\% no se confirmó la etiología (diagnóstico clínico).

La prevalencia de Diabetes Mellitus entre los casos de TB mayores de 15 años de la Región Metropolitana fue de 15,6\% (IC95\% = 13,2$18,2)$. La prevalencia de DM entre los hombres con TB fue de $13,4 \%$ y entre las mujeres con TB de $19,1 \%$ (RP 0,7; IC95\% 0,5-0,9). La prevalencia de DM entre los casos de TB por Servicio de Salud se observa en la Tabla 1.

$\mathrm{Al}$ ajustar la prevalencia de DM entre los casos de TB analizados por edad, ésta corresponde a
12,44\% (IC95\% = 12,42-12,5) con una razón de prevalencia (RP) de 1,29 (IC 95\% 1,28-1,29) respecto a la población general. Si se realiza el ajuste por sexo, la prevalencia de DM entre los casos de TB sería de 16,3\% (IC95\%=16,2-16,3) con una $\mathrm{RP}$ de 1,73 (IC95\% = 1,72-1,73) respecto a la población general.

La prevalencia de DM en casos de TB según grupo de edad se observa en la Tabla 2. Si bien para todos los grupos de edad la prevalencia de diabetes es mayor en el grupo de pacientes con tuberculosis que en la población general, esta diferencia no es estadísticamente significativa.

La prevalencia de DM en casos de TB según sexo comparado con población general se observa en la Tabla 3. Para ambos grupos, y más para las mujeres, la prevalencia de diabetes es mayor que en la población general, valores que tienen significancia estadística.

La incidencia estimada de TB entre la población con DM mayor de 15 años de la Región Metropolitana para el año 2012 corresponde a 24,3 por 100.000 , y la incidencia de TB en la población no diabética mayor de 15 años de la región metropolitana fue de 13,7 por 100.000

Tabla 1. Prevalencia de diabetes mellitus en casos nuevos de tuberculosis mayores de 15 años por Servicio de Salud. Región Metropolitana. 2012

\begin{tabular}{|lcr|}
\hline Servicio de Salud & $\begin{array}{c}\text { Prevalencia } \\
\text { DM (\%) } \\
\text { n }=\mathbf{8 2 1}\end{array}$ & IC 95\% \\
\hline Metropolitano Norte & 12,3 & $7,5-19,7$ \\
\hline Metropolitano Central & 11,5 & $7,7-16,9$ \\
\hline Metropolitano Sur & 18,7 & $13,5-25,4$ \\
Metropolitano oriente & 18,9 & $11,6-29,2$ \\
\hline Metropolitano Occidente & 13,9 & $9,4-19,9$ \\
\hline Metropolitano Sur oriente & 21,2 & $14,7-29,6$ \\
\hline
\end{tabular}

Tabla 2. Prevalencia de Diabetes mellitus en casos de Tuberculosis según grupo de edad. Región Metropolitana. 2012

\begin{tabular}{|lccc|}
\hline Grupo de edad & $\begin{array}{c}\text { Prevalencia DM en casos } \\
\text { TB (\%) } \\
\mathbf{n = 8 2 1}\end{array}$ & $\begin{array}{c}\text { Prevalencia de DM (\%) } \\
\text { según ENS** } \\
\mathbf{n}=\mathbf{4 . 6 5 6}\end{array}$ & $\begin{array}{c}\text { Razón de Prevalencia } \\
\text { (IC 95\%) }\end{array}$ \\
\hline 15-24 años & 0,98 & 0,4 & $5,1 \quad(0,3-69)$ \\
\hline 25-44 años & 6,3 & 3,8 & $1,66(0,98-2,8)$ \\
\hline 45-64 años & 20,7 & 16,9 & $1,2(0,95-1,58)$ \\
65 y más años & 30,9 & 25,8 & $1,19(0,9-1,5)$ \\
\hline
\end{tabular}

*ENS = Encuesta Nacional de Salud. 
Tabla 3. Prevalencia de Diabetes mellitus en casos de Tuberculosis según sexo.

Región Metropolitana. 2012

\begin{tabular}{|lccc|}
\hline Sexo & $\begin{array}{c}\text { Prevalencia DM en casos } \\
\text { TB (\%) } \\
\mathbf{n = 8 2 1}\end{array}$ & $\begin{array}{c}\text { Prevalencia de DM según } \\
\text { ENS (\%) } \\
\mathbf{n}=\mathbf{4 . 6 5 6}\end{array}$ & $\begin{array}{c}\text { Razón de Prevalencia } \\
(\text { IC 95\%) }\end{array}$ \\
Hombres & 13,4 & 8,4 & $1,6(1,2-2,0)$ \\
Mujeres & 19,1 & 10,4 & $1,8(1,3-2,4)$ \\
\hline
\end{tabular}

habitantes este mismo año. Por lo tanto la incidencia de TB entre los diabéticos es un $70 \%$ más que la de la población sin diabetes en la Región Metropolitana.

\section{Discusión}

En este estudio se ha encontrado una asociación entre TB y DM que es inferior a lo planteado por la OMS y por diversos estudios internacionales. En Chile, un diseño de casos y controles realizado por Ramonda et al, que estudió los casos de TB del año 2006 al 2009 en el Servicio de Salud Metropolitano Sur, obtuvo una fuerte asociación entre ambas patologías (OR 2,3 ajustado por edad y nivel socioeconómico) con características de los casos de TB con diabetes en cuanto a edad y sexo similares con lo reportado por este estudio 9 .

Una de las limitaciones del estudio corresponde al hecho de que la medición de la variable diabetes mellitus se hizo a través de lo registrado en SIGGES y no directamente con exámenes de laboratorio, por lo que pueden existir casos de tuberculosis a quienes no se les ha realizado el diagnóstico de diabetes o bien que no estén registrados en el sistema. También podrían existir diabéticos enfermos de tuberculosis en etapas precoces a quienes aún no se les ha hecho el diagnóstico.

Por otra parte, por ser un estudio transversal, en el que no se determinó cuál de las dos patologías se diagnosticó primero, la dirección de la asociación entre diabetes y tuberculosis no queda claramente establecida. La declinación de la respuesta inmune que produce la diabetes mellitus se ha establecido como un factor de riesgo para el desarrollo de infecciones como es la tuberculosis ${ }^{9}$ lo que significaría que la diabetes constituye la exposición y la tuberculosis el efecto. Sin embargo, la relación de ambas patologías es más compleja, ya que la tuberculosis activa también aumenta los niveles de glicemia por tratarse de un proceso de inflamación crónica ${ }^{10}$. Sin embargo, esta causalidad reversa ha sido descrita para una minoría de pacientes (menos del $4 \%)^{9}$, por lo que nos atrevemos a señalar que las mayores prevalencias de diabetes en casos de tuberculosis en relación a población general se deben al mayor riesgo de los diabéticos de desarrollar una tuberculosis activa.

Si bien entre los casos de TB de la Región Metropolitana la relación hombre: mujer corresponde a lo observado en la epidemiología de la tuberculosis en Chile, en que los hombres constituyen $2 / 3$ del total de $\operatorname{casos}^{11}$, en el caso de los diabéticos esta relación se estrecha a casi $50 \%$ para cada sexo, con una prevalencia mayor de diabetes en las mujeres con TB respecto a los hombres, lo que concuerda con los hallazgos de la $\mathrm{ENS}^{7}$, pero también en relación a las mujeres de la población general. Por lo tanto, el peso de la diabetes como factor de riesgo entre las mujeres con tuberculosis es mayor que para los hombres.

La incidencia de tuberculosis aumenta con la edad, así como la diabetes mellitus es más prevalente a mayor edad ${ }^{7,11}$, por lo que la edad puede actuar como variable de confusión. Al ajustar por edad la tasa de prevalencia para el cálculo de la RP se evidencia que efectivamente la mayor de edad de los casos de tuberculosis influye en la mayor prevalencia de diabetes, pero aún existe casi un 30\% de mayor probabilidad de tuberculosis entre diabéticos que no se explica por la edad. Sin embargo, la estratificación por grupo de edad no mostró resultados estadísticamente significativos. Sería pertinente poder realizar la comparación de las prevalencias de DM con la población de la Región Metropolitana, sin embargo, no pudimos obtener estos datos diferenciados por rango de edad.

Otro hallazgo destacable de este estudio corresponde a las diferencias en la prevalencia de DM en casos de TB entre los distintos Servicios de Salud, siendo el Metropolitano Sur Oriente el que tiene la mayor prevalencia y el Metropolitano Central, la menor. Sería interesante poder comparar estas cifras con la prevalencia de DM entre la población de estos Servicios de Salud, dato que tampoco se pudo obtener.

Según la ENS 2010, la DM se concentra en 
estratos socioeconómicos bajos ${ }^{7}$, y es conocida la asociación de existe entre TB y pobreza, por lo que el análisis del estrato socioeconómico, que no ha sido considerado en este estudio, pudiese influir en sus resultados.

Finalmente, si bien Chile se encuentra en fase de eliminación de la tuberculosis, la prevalencia de la diabetes ha ido aumentando en la población, por lo que es probable que su importancia como factor de riesgo para TB vaya también en aumento. Esto implica que es de suma importancia incentivar a los equipos de salud a registrar la diabetes como factor de riesgo en los registros normados del Programa de Tuberculosis, ya que como se observó a la luz de este estudio, prácticamente no había ningún caso de diabetes registrado entre los factores de riesgo. Además, consideramos importante ampliar los estudios en la población diabética a nivel nacional para confirmar si la situación de la Región Metropolitana se reproduce.

\section{Conclusiones}

El estudio demuestra que los diabéticos constituyen un grupo de riesgo para tuberculosis, especialmente entre las mujeres, por lo que las estrategias de focalización para la localización de casos deberán contemplar a este grupo como prioritario.

\section{Bibliografía}

1.- HERRERA T. La situación de la tuberculosis en Chile y los actuales desafíos. Visita de la OPS al Programa de Control de la Tuberculosis de Chile. Rev Chil Enf Respir 2013; 29: 46-9.

2.- MINISTERIO DE SALUD. Estrategia Nacional de Salud para el cumplimiento de los Objetivos Sanitarios de la Década 2011-2020. Santiago. 2011

3.- GARCÍA C. Tuberculosis en grupos de riesgo en la Región Metropolitana, 2008. Rev Chil Enf Respir 2010; 26: 105-11.

4.- PROGRAMA NACIONAL DE CONTROL DE LA TUBERCULOSIS. Manual de Organización y Normas Técnicas. 2005. Ministerio de Salud.

5.- WORLD HEALTH ORGANIZATION. Collaborative framework for care and control of tuberculosis and diabetes [Libro electrónico]. Geneva: WHO Document Production Service; 2011 [Consultado: 31 de agosto de 2013]. Disponible en http://whqlibdoc.who.int/publications/2011/9789241502252_eng.pdf

6.- SOLIS M, AGUIRRE C, GODORECCI B, MOIS Y, ROJAS F, JIMÉNEZ L. Prevalencia de Diabetes Mellitus en Chile. Revista Asociación Latinoamericana de Diabetes [Articulo en línea]. [Fecha de consulta: 30 de agosto de 2013]. Disponible en: http://www.revistaalad. com.ar/website/articulo.asp?id=67\&pagina $=5$

7.- MINISTERIO DE SALUD. Encuesta Nacional de Salud ENS Chile 2009-2010.

8.- PROGRAMA NACIONAL DE CONTROL Y ELIMINACIÓN DE LA TUBERCULOSIS. Registro Nacional de Tuberculosis. Ministerio de Salud.

9.- RAMONDA P, PINO P, VALENZUELA L. Diabetes mellitus como factor predictor de tuberculosis en el Servicio de Salud Metropolitano Sur en Santiago, Chile. Rev Chil Enf Respir 2012; 28: 277-85.

10.- AGUILAR S. Diabetes y tuberculosis: en el laberinto del subdesarrollo. Rev Invest Clin 2005; 57: 82-4.

11.- INFORME SITUACIÓN DE LA TUBERCULOSIS EN CHILE 2008-2011. Programa Nacional de Control y Eliminación de la Tuberculosis. Ministerio de Salud.

Correspondencia a:

Dra. Tania Herrera M.

Directora del Programa de Control y Eliminación

de la Tuberculosis.

Ministerio de Salud. Chile.

Email: tania.herrera@minsal.cl 